\title{
A review on the technical and dosimetric aspects of stereotactic body radiation therapy (SBRT)
}

\author{
Cheng B Saw $\cdot$ Shanglian Bao $\cdot$ Sicong Li
}

Received: 17 February 2012 /Accepted: 16 April 2012 / Published online: 2 May 2012

(C) Springer-Verlag 2012

\begin{abstract}
Stereotactic body radiation therapy (SBRT) or stereotactic ablative radiotherapy is currently enjoying much popularity due to its favorable outcomes. This treatment option had been derived from stereotactic radiosurgery (SRS) which uses focus irradiation technique to deliver a very high dose to a small intracranial target. The focus irradiation technique is performed using multiple collimated beams directed at the target from around the patient. Advancement in technology has provided similar tools on tomotherapy, modern medical linear accelerators, and dedicated system such as the Cyberknife to perform SBRT. Such tools include immobilization systems, 3D treatment planning systems, image-guidance system for precise localization, and accurate dose delivery systems. Each of these systems is being briefly reviewed. While the technical specifications of the equipment for SBRT are favorable, the dosimetric aspects need attention when performing treatment planning and managing target/organ motion. Because of the delivery of high doses in one or few fractions, special
\end{abstract}

\section{B. Saw $(\bowtie)$}

Northeast Radiation Oncology Centers (NROC),

CBSaw Publishing, LLC,

Harrisburg, PA, USA

e-mail: cbsaw2003@yahoo.com

\section{S. Bao}

Research Center for Tumor Diagnosis and Therapeutic Physics, Peking University,

Beijing, China

S. Li

Department of Radiation Oncology,

University of Nebraska Medical Center,

Omaha, NE, USA considerations must be given to transient doses through neighboring structures to avoid or reduce radiation toxicities for SBRT. Like SRS, direct involvement of medical physicists is essential for both the quality assurance and clinical procedures.

Keywords SBRT $\cdot$ SABR $\cdot$ Safety $\cdot$ Planning $\cdot$ SRS . Cyberknife

\section{Introduction}

Stereotactic body radiation therapy (SBRT), also known as stereotactic ablative radiotherapy is an emerging treatment option that uses highly focused irradiation technique to treat extracranial lesions. The use of highly focused irradiation technique is not new but derived from stereotactic radiosurgery (SRS) used for treating intracranial lesions under the guidance of a stereotactic frame system. SBRT is defined by the American Society for Radiology Oncology (ASTRO) and American College of Radiology as the very precise delivery of a high radiation dose to an extracranial target within the body using either a single dose or a small number of fractions [1]. Radiobiologically, the high-dose fraction regimens will yield more potent biological effects. For example, the delivery of $60 \mathrm{~Gy}$ in 30 fractions and in three fractions will yield a biological equivalent dose of 72 and $180 \mathrm{~Gy}$, respectively, making it highly beneficial in the treatment of radio-resistant tumors such as non-small cell lung cancers [2]. Literature reviews have shown that the principal application of SBRT has been in the treatment of lung, liver, pancreas, kidney, prostate, and spine [3, 4].

As an emerging treatment option, SBRT is currently enjoying popularity with favorable outcomes. The 
transformation of radiation oncology practice to imagebased paradigm has provided similar tools on modern medical linear accelerators to those used in the focused irradiation technique. This has led to the widespread implementation of SBRT treatment technique. However, it must be emphasized that SBRT is a specialized form of radiation therapy that must adhere to stringent technical requirements for precise delivery of high doses in one or few fractions. The objective of this article is to review the physical and dosimetric requirements for the successful implementation of SBRT.

\section{Stereotactic radiosurgery principles}

SRS was developed for a single-fraction stereotactic irradiation which is conceptually different from conventional fractionated radiation therapy. In SRS, the intent is the delivery of high dose striving towards the ablative tumor effect compared with the fractionated technique of seeking normal tissue recovery in conventional radiation therapy. Because of the high biological potency, the delivery of the radiation dose is guided using a stereotactic frame system for precise irradiation of the target volume to minimize radiation injury. Stereotactic frame system allows the assignment of three-dimensional (3D) coordinates to deepseated brain lesions or neuroanatomical structures relative to the system. This relationship is established by scanning a rigid stereotactic frame system that is fixed to the skull. Lars Leksell, a Swedish neurosurgeon was the first to combine the two developing fields of stereotaxy and radiation therapy and introduced the term "radiosurgery" as a non-invasive procedure of radiosurgically removing the lesion in 1951 [5]. The Gamma Knife is a dedicated unit designed by Leksell for the specific purpose of performing stereotactic radiosurgery to the brain. The latest Gamma Knife model has cobalt-60 sources distributed in a circular array in a

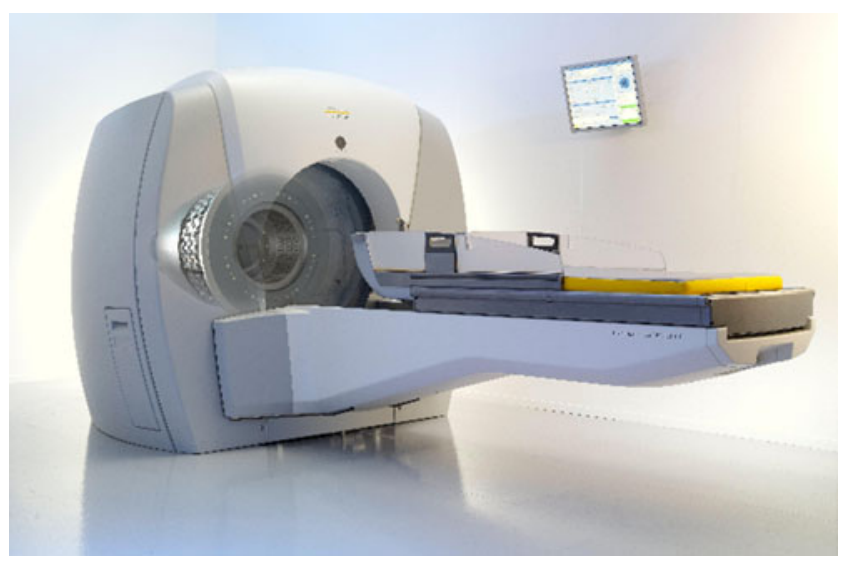

Fig. 1 Latest Gamma Knife model (Perfexion) heavily shielded assembly producing 192 highly collimated narrow beams that focus on the tumor as a means of minimizing the doses to surrounding brain tissues (see Fig. 1). The 1980s saw the adaptation of medical linear accelerators (linacs) for intracranial stereotactic dose delivery, again using rigid stereotactic head frame and specialized treatment planning system [6]. Multiple arcs are used in linac-based radiosurgery as a means of simulating the multiple collimated beams as the gantry rotates around the patient (see Fig. 2). The patient immobilization and set-up rely on the rigid attachment of the stereotactic head frame either at the treatment couch or through a stand that is attached to the base of the medical linear accelerator. Because of the complexity of the procedure, SRS are performed at a limited number of institutions with direct involvement of the medical physicists.

\section{Technological advancement in SBRT}

Existing dose delivery systems such as tomotherapy units and modern medical linear accelerators designed for the implementation of IMRT have the same tools necessary for the implementation of the SBRT treatment technique. These include effective patient immobilization system, 3D treatment planning systems, image-guidance systems (IGRT) for precise target localization, accurate treatment delivery systems, and the ability to produce a steep isodose gradient outside the target volume (see Fig. 3). The steepness of the isodose gradient can be measured by taking the ratio of the volume receiving $50 \%$ of the prescription dose to the planning target volume [7]. Modern medical linear accelerators are much improved having tighter isocenter tolerance to less than $1 \mathrm{~mm}$. The movement of the digital treatment couch in three dimensions can be determined to within $1 \mathrm{~mm}$. Coupled with image-guidance system, the dose delivery system can position a patient precisely to within a millimeter. Like SRS, multiple arcs or many static fields are used for the implementation of SBRT. The design of dose delivery system and the incorporation of IGRT have led to the introduction of Cyberknife, a system designed for radiosurgery. The system has a compact linear accelerator mounted on a robotic arm that delivers radiation dose from around the patient as shown in Fig. 4. By design, numerous small beams are needed to deliver the prescribed dose to the patient as shown in Fig. 5. The Novalis is a medical linear accelerator modified to perform focused irradiation technique. Finer multileaf collimation systems with smaller leaf width are also becoming more accessible on modern medical linear accelerators. The precise targeting is performed using IGRT. In IGRT, the patient is imaged using planar imaging or tomographic imaging prior to dose delivery. 
Fig. 2 Multiple arcs used in linac-based radiosurgery

These image datasets are fused to the image datasets taken for treatment planning to determine the relative alignment. The fusion of image datasets can be performed manually or automatically based on a number of fusion algorithms [8]. The fused images as shown in Fig. 6 provide the mismatches between the patient's position prior to treatment to that at the time of treatment planning. These mismatches allow for couch
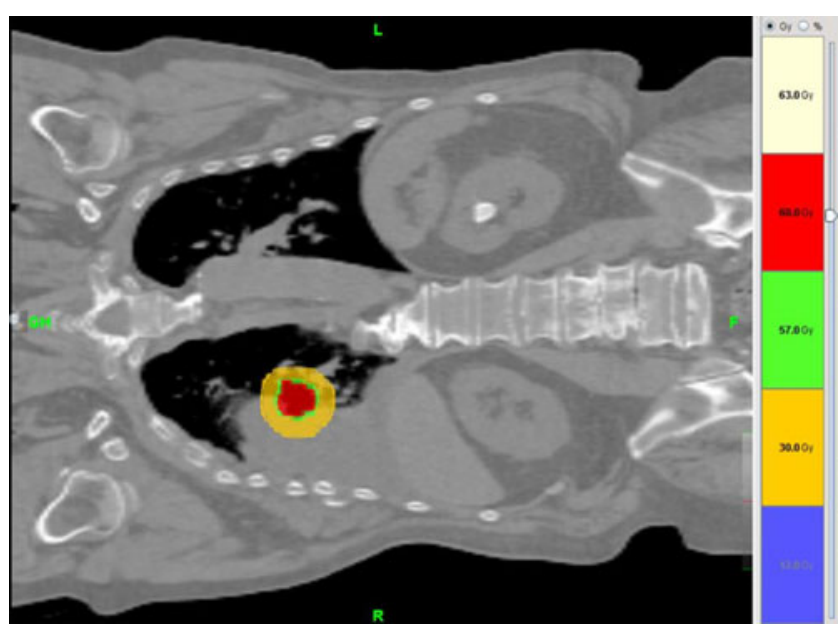

Fig. 3 SBRT dose distribution movement for precise patient positioning and hence precise beam targeting. In using the same tools as offered by advanced dose delivery systems, it is generally believed that any dose delivery system that can perform IMRT with IGRT capabilities can also be used to perform SBRT.

Unlike the immobilization for intracranial treatment, extracranial region does not have rigid bony landmarks for the

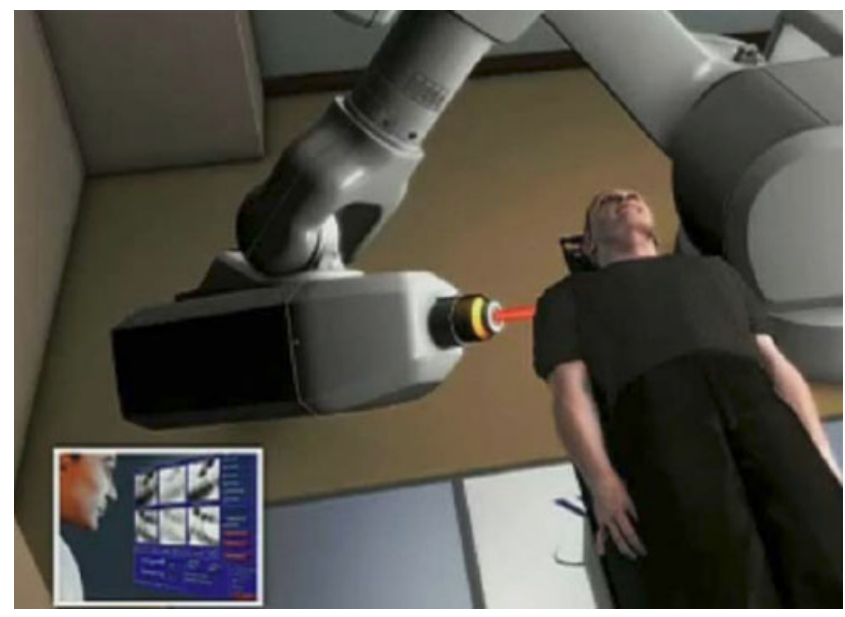

Fig. 4 Compact linear accelerator mounted on a robotic arm delivering radiation dose from around the patient 


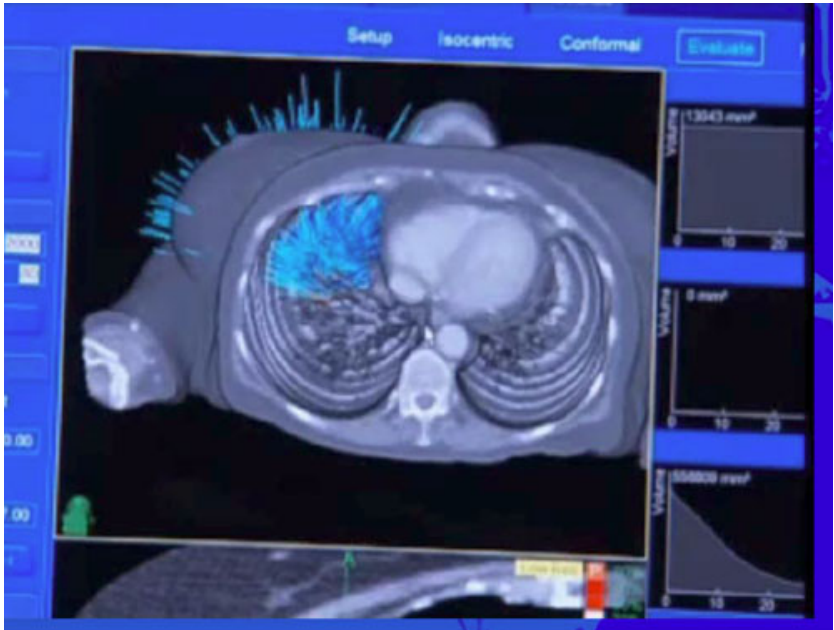

Fig. 5 Numerous small beams needed to deliver the prescribed dose to the patient using Cyberknife

physical fixation of the stereotactic device. Furthermore, the trend in radiation therapy has been towards non-invasive procedure and frameless technology. Lax et al. [9] developed a stereotactic body frame which together with a vacuum bag immobilized the patient from the head to the midthigh. Many stereotactic systems used today for SBRT use a similar set-up with body frame immobilization technique as shown in Fig. 7. However, the position of the tumor generally does not correlate rigidly to the external body contour compared with those in the brain.

The ability to manage tumor/patient motion has significantly facilitated the precise delivery of radiation dose in SBRT. The introduction of fast CT scanners has allowed the ability to perform 4D-CT scanning. Such image dataset allows the tracking of the target in particular lung tumors throughout the breathing cycle. This invaluable information on the extent of the tumor motion permits the delineation of

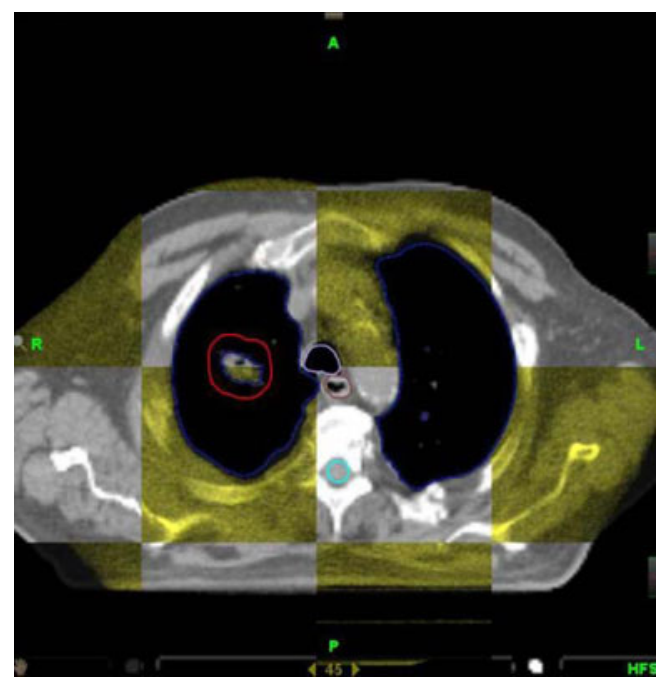

Fig. 6 Fusion of positioning and planning image datasets

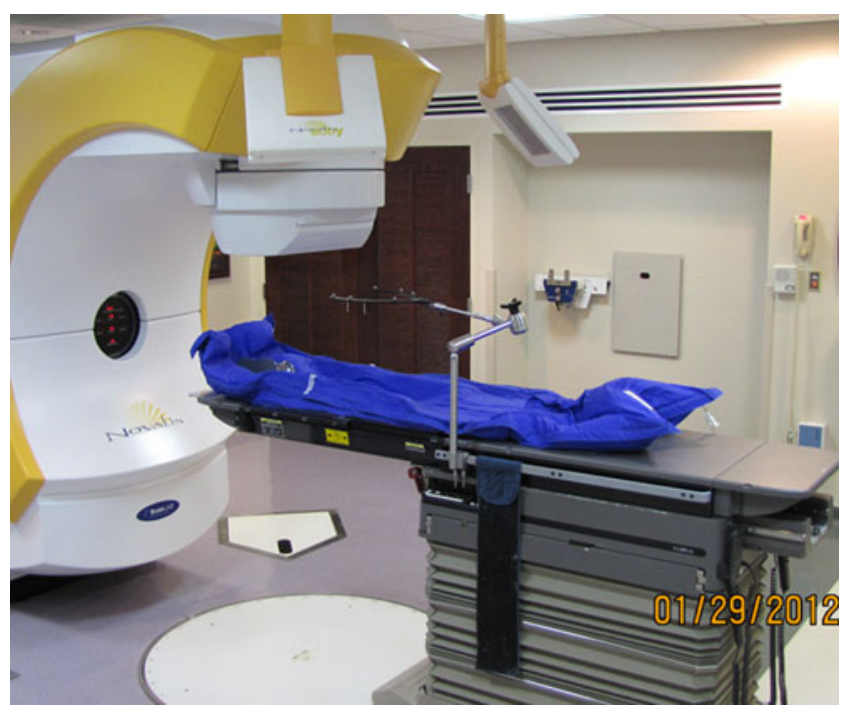

Fig. 7 Body frame immobilization system

internal target volume (ITV) for irradiation (as shown Fig. 8) based on the phases of the breathing cycle. Beside using motion tracking technique, force-breathing technique using abdominal compression device as shown in Fig. 9 and breath-hold technique limits breathing motion and hence the tumor motion. A review of the respiratory gating technology had been published by Saw et al. [10]

\section{Treatment planning}

The introduction of 3D treatment planning systems has accelerated the implementation of SBRT. 3D treatment planning systems also known as image-based treatment planning systems incorporates $\mathrm{CT}$ and other imaging modalities such as MRI and PET to create virtual patients for treatment

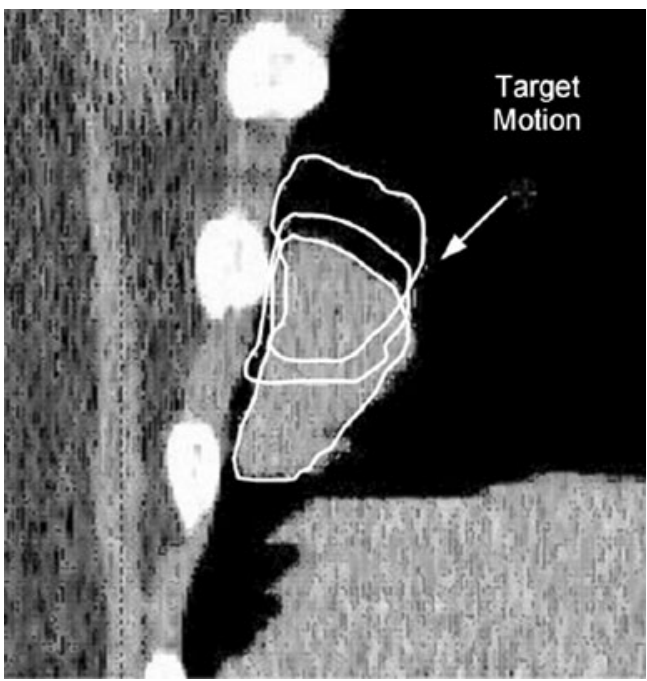

Fig. 8 Delineation of internal target volume (ITV) for irradiation 


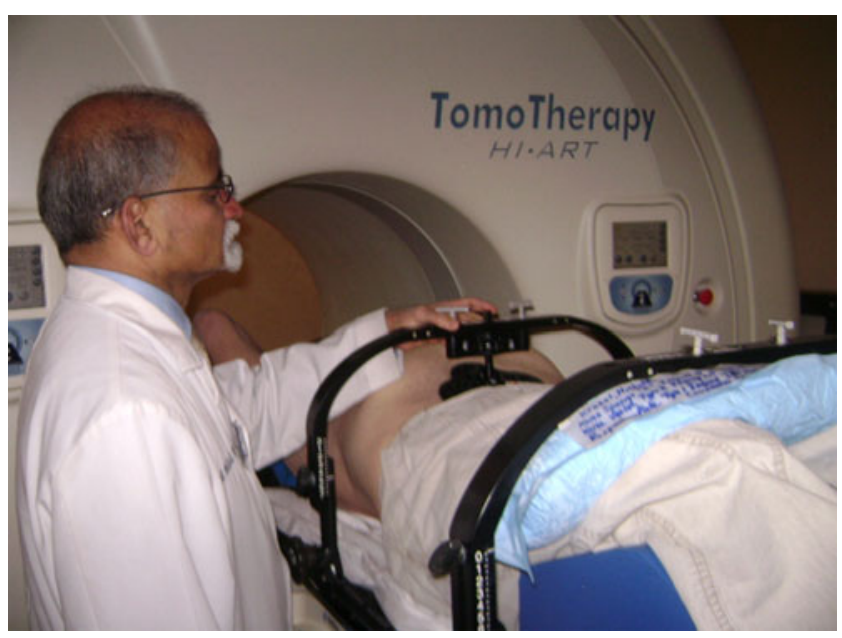

Fig. 9 Force-breathing technique

planning. The anatomical information from CT or MRI images allows for the precise delineation of the tumors relative to the patient's anatomy. In addition, the CT information allows for the incorporation of tissue inhomogeneity correction into the dose calculation algorithms to improve the accuracy of dose determination in the media that is different from water in particular in the lung. The visualization of the patient anatomy in three dimensions allows for better beam placements that minimizes or avoid doses through radiation sensitive anatomical structures. 3D treatment planning systems are required for the implementation of complex treatment techniques of conformal radiation therapy (CRT) and intensity-modulated radiation therapy (IMRT). These treatment techniques require higher level of precision in patient positioning carried out using IGRT technology.

There is the possibility of misunderstanding between the approaches of SBRT and IMRT. IMRT uses few fields with beam modulation to improve the conformality. The extension on the use of a few fields had led to increased transient doses resulting in unfavorable high level of radiation toxicities for SBRT. Publications and educational symposia have demonstrated that the use of limited number of fields produces severe skin toxicities [11-15]. It is of paramount importance to design treatment plan having a significant number of fields. The minimum number of fields needed for a successful SBRT treatment is dependent on the normal tissue dose tolerances. While the tolerance doses in the brain are well known, this is not the case for organs in other part of the body for hypo-fractionated treatments. It is therefore wise to exhibit extraordinary care on the dose limits for neighboring critical organs to the lesions when designing individualized treatment plans to prevent radiation toxicities. There are a number of articles reporting on the radiation injuries after SBRT $[16,17]$. The common radiation toxicities reported in the treatment of lung are (a) radiation pneumonitis, (b) rib fracture and pain, (c) esophageal toxicity, (d) brachial plexopathy, and (e) bleeding.

\section{Safety}

Patient safety has become a critical concern as technological advancements have been so rapid that safeguards and quality assurance are not keeping in pace [18]. Recent articles in the New York Times highlighted the importance of quality assurance of the equipment as well as the clinical procedures [19-22]. Small errors in positioning can lead to a detrimental outcome both in term of patient cure and radiation injury [20-22]. The American Association of Physicists in Medicine and ASTRO have published documents on the quality assurance aspects of SBRT [23, 24]. The quality assurance implemented should check the integrity of (a) the moving mechanism of the dose delivery systems such as isocenter test and treatment table motion, (b) the image quality and spatial resolution of the imaging systems, (c) motion-gating mechanisms, and (d) treatment planning systems in addition to the typical routine daily checks on the radiation dose delivery systems.

Lastly, the success of SBRT is very dependent on the experience level of the referring physician (neurosurgeon in the case of spine irradiation), radiation oncologist and the expertise of the physics team to perform quality assurance and the design of the treatment plans that minimize doses to critical organs. Transient doses to critical organs have been shown to significantly impact on the outcome of the treatments. While the technology appears to be accessible on modern medical linear accelerators, the stringent requirements must be assessed prior to the implementation of SBRT including the (a) imageguidance dose delivery systems, (b) treatment planning systems, and (c) the expertise of the medical physics team.

\section{Conclusions}

It must be emphasized that SBRT is a specialized form of radiation therapy and has stringent requirements for quality assurance and treatment planning compared with CRT and IMRT treatment techniques. Because of the high dose delivered in one or few fractions, experience in treatment planning to generate an optimal individualized treatment plan is very critical to avoid or reduce radiation toxicities. The awareness of transient doses through various critical anatomical structures and their dose limits is critical. In order to enjoy the success of SBRT, any radiation oncology practice wishing to implement this treatment option must have an experienced medical physics team knowledgeable in focused irradiation technique to handle safeguards and quality assurance for the precise dose delivery. Like SRS, direct involvement of medical physicists is essential. 
Conflicts of interest None. There is no participation in vendor's interest.

\section{References}

1. Potters L, Kavanagh B, Galvin JM et al (2010) American Society for Therapeutic Radiology and Oncology (ASTRO) and American College of Radiology (ACR) practice guideline for the performance of stereotactic body radiation therapy. Int J Radiat Oncol Biol Phys 76:326-332

2. Teh BS (2007). Image-guided stereotactic body radiation therapy (SBRT): an emerging treatment paradigm with a new promise in radiation oncology. Biij 1/e5/2007. Available at http://www. biij.org/2007/1/e5/

3. Tipton K, Launders JH, Inamder R et al (2011) Stereotactic body radiation therapy: scope of the literature. Ann Intern Med 154:737-745

4. Martin A, Gaya A (2010) Stereotactic body radiotherapy: a review. Clin Oncol 22:157-172

5. Leksell LT (1951) The stereotactic method and radiosurgery of the brain. Acta Chir Scand 102:316

6. AAPM (1995) AAPM report no. 54. Stereotactic radiosurgery. AIP, Woodbury, NY

7. Diot Q, Kavanagh S, Timmerman R et al (2012) Biological-based optimization and volumetric modulated arc therapy delivery for stereotactic body radiation therapy. Med Phy 39:237-245

8. Saw CB, Chen H, Beatty RE et al (2008) Multimodality image fusion and planning and dose delivery for radiation therapy. Med Dosm 33:149-155

9. Lax I, Blomgren H, Naslund I et al (1994) Stereotactic radiotherapy of malignancies in the abdomen: methodological aspects. Acta Oncol 33:677-683

10. Saw CB, Brandner E, Selvaraj R, et al (2007) A review on the clinical implementation of respiratory-gated radiation therapy. Biij 1/e40/2007. Available at http://www.biij.org/2007/1/e40/

11. Hoppe BS, Laser BL, Kowalski AV et al (2008) Acute skin toxicity following stereotactic body radiation therapy for stage I non-smallcell lung cancer: who's at risk? Int J Radiat Oncol Biol Phys $72: 1283-1286$
12. Saw CB (2010). Stereotactic body radiation therapy (SBRT). Available at http://www.medicaldosimetry.org/meetings/2010pre sentations/6-Saw-SBRT.pdf

13. Timmerman R (2009). Application of hypofractionation in the lung. Available at http://www.aapm.org/meetings/amos2/pdf/296984-96276-902.pdf

14. Kavanagh BD (2009). Radiation biology \& future trends of SBRT. Available at http://www.aapm.org/meetings/amos2/pdf/42-1214345768-976.pdf

15. Kavanagh BD (2009). Educational course TU-A-BRB-1. Available at http://www.aapm.org/meetings/amos2/pdf/49-1445286086-963.pdf

16. Linda A, Trovo M, Bradley JD (2011) Radiation injury of the lung after stereotactic body radiation therapy (SBRT) for lung cancer: a timeline and pattern of CT changes. Eu J Radiol 79:147-154

17. Milano MT, Constine LS, Okunieff P. Normal tissue toxicity after small field hypofractionated stereotactic body radiation. Rad Oncol 3:36; 2008. Available at http://www.ro-journal.com/content/pdf/ 1748-717X-3-36.pdf

18. Saw CB. Patient safety in radiation medicine. Available at http:// imaging-radiation-oncology.advanceweb.com/ebook/magazine. aspx?EBK=IR091911\#

19. Radiation offers new cures, and ways to do harm. New York Times $2010 \mathrm{http} / /$ www.nytimes.com/2010/01/24/health/24radiation.html? pagewanted $=$ all

20. A pinpoint beam strays invisibly, harming instead of healing. http://www.nytimes.com/2010/12/29/health/29radiation.html? pagewanted=all

21. Radiation errors reported in Missouri. New York Times 2010 http://www.nytimes.com/2010/02/25/us/25radiation.html? scp $=43 \&$ sq= bogdanich\&st $=$ cse

22. As technology surges, radiation safeguards Lag. New York Times $2010 \mathrm{http} / / /$ www.nytimes.com/2010/01/27/us/27radiation.html? $\mathrm{scp}=20 \&$ sq $=$ bogdanich \&st $=$ cse \&pagewanted $=3$

23. Benedict SH, Yenice KM, Followill D et al (2010) Stereotactic body radiation therapy: the report of the AAPM Task Group 101. Med Phys 37:4078-4101

24. Solberg TD, Balter JM, Benedict SH et al (2012) Quality and safety considerations in stereotactic radiosurgery and stereotactic body radiation therapy: executive summary. Pro 2:2-9 